\title{
Monolithic Millimeter-Wave and Picosecond Electronic Technologies
}

Wilson K. Talley

Neville C. Luhmann

\section{RECEIVED \\ MAY 211996 \\ OSTI}

March 12, 1996

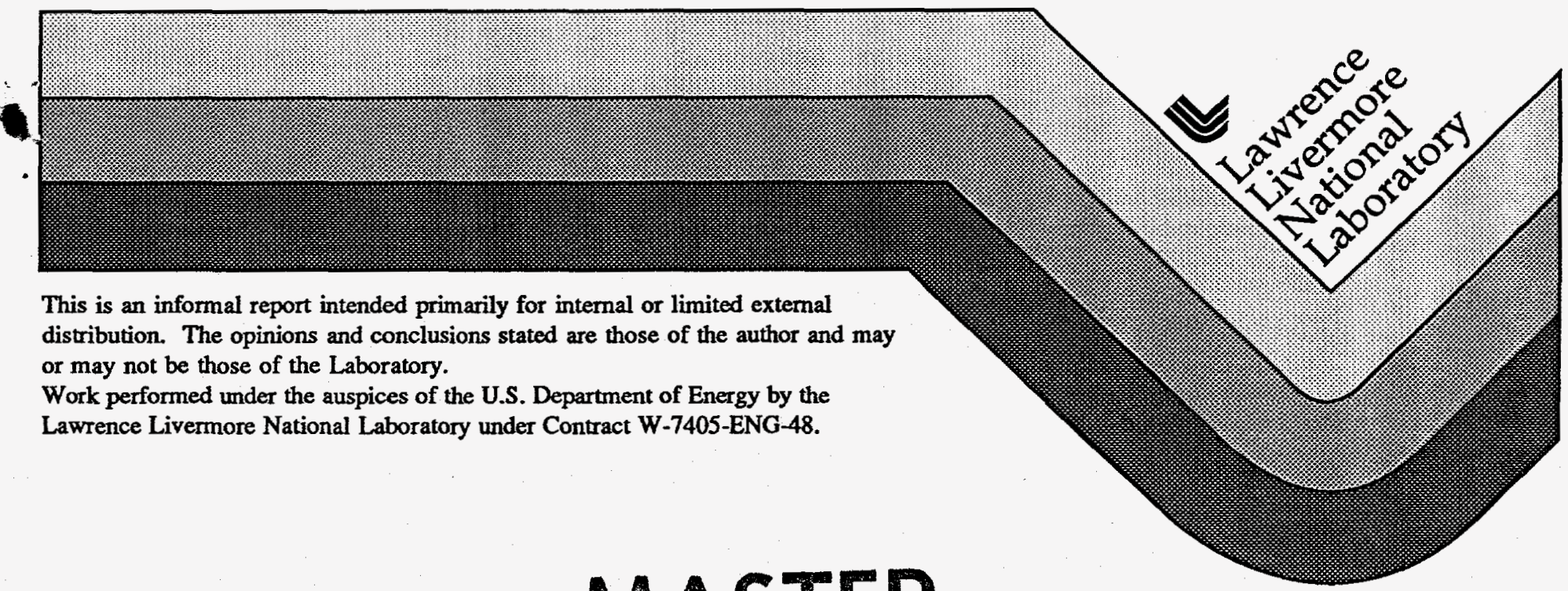




\section{DISCLAMER}

This document was prepared as an account of work sponsored by an agency of the United States Government. Neither the United States Covernment nor the University of California nor any of their employees, makes any warranty, express or implied, or assumes any legal liability or responsibility for the accuracy, completeness, or usefulness of any information, apparatus, product, or process disclosed, or represents that its use would not infringe privately owned rights. Reference herein to any specific commercial product, process, or service by trade name, trademark, manufacturer, or otherwise, does not necessarily constitute or imply its endorsement, recommendation, or favoring by the United States Government or the University of California. The views and opinions of authors expressed herein do not necessarily state or reflect those of the United States Government or the University of California, and shall not be used for advertising or product endorsement purposes.

This report has been reproduced directly from the best available copy.

Available to DOE and DOE contractors from the Office of Scientific and Technical Information P.O. Box 62, Oak Ridge, TN 37831

Prices available from (615) 576-8401, FTS 626-8401

Available to the public from the National Technical Information Service

U.S. Department of Commerce 5285 Port Royal Rd. Springfield, VA 22161 


\section{DISCLAIMER}

Portions of this document may be illegible in electronic image products. Images are produced from the best available original document. 


\title{
MONOLITHIC MILLIMETER-WAVE AND PICOSECOND ELECTRONIC TECHNOLOGIES
}

\author{
Wilson K. Talley \\ Lawrence Livermore National Laboratory, University of California \\ Livermore, California 94550 \\ Neville C. Luhmann \\ Department of Applied Science, University of California at Davis \\ Livermore, California 94550
}

March 12, 1996

\begin{abstract}
Theoretical and experimental studies into monolithic millimeter-wave and picosecond electronic technologies have been undertaken as a collaborative project between the Lawrence Livermore National Laboratory (LLNL) and the University of California Department of Applied Science Coherent Millimeter-Wave Group under the auspices of the Laboratory Directed Research and Development Program at LLNL. The work involves the design and fabrication of monolithic frequency multiplier, beam control, and imaging arrays for millimeter-wave imaging and radar, as well as the development of high speed nonlinear transmission lines for ultra-wideband radar imaging, time domain materials characterization and magnetic fusion plasma applications. In addition, the Coherent Millimeter-Wave Group is involved in the fabrication of a state-of-the-art X-band ( 8-11 $\mathrm{GHz}$ ) RF photoinjector source aimed at producing psec high brightness electron bunches for advanced accelerator and coherent radiation generation studies.
\end{abstract}




\section{Introduction}

The Monolithic Millimeter-Wave and Picosecond Electronic Technologies Initiative is designed to advance the state-of-the-art in solid state sources for both transmitters and receivers together with imaging receiver arrays. The work involves the design and fabrication of monolithic frequency multiplier, beam control, and imaging arrays for millimeter-wave imaging and radar; as well as the development of high speed nonlinear transmission lines for ultra-wideband radar imaging, time domain materials characterization and magnetic fusion plasma applications. Other important uses for DOE and other national applications include detection of weapons and contraband, all-weather imaging radars, automotive collision avoidance radar, radio astronomy, atmospheric radiometry, nondestructive testing, robotic vision, space based radar, planetary mapping and resource evaluation, direct satellite broadcasting, and secure communications.

\section{Millimeter-Wave Imaging Arrays}

Multichannel hybrid and monolithic Schottky diode mixer arrays were under investigation for millimeter-wave imaging applications. A 20 channel hybrid array, designed to collect second harmonic ECE radiation over a bandwidth of 90-120 GHz, was fabricated and installed on the TEXT-U tokamak in Austin, TX in FY96. The TEXT-U imaging system, as illustrated in Fig. 1, consists of a pair of high density polyethylene focusing lenses which image the plasma electron cyclotron emission onto the array. Plasma emissions are measured heterodyne by mixing the collected radiation with a local oscillator (LO) signal fed optically from the reverse side. Incident radiation from the plasma is coupled into the substrate side through a hyper-hemispherical substrate lens.

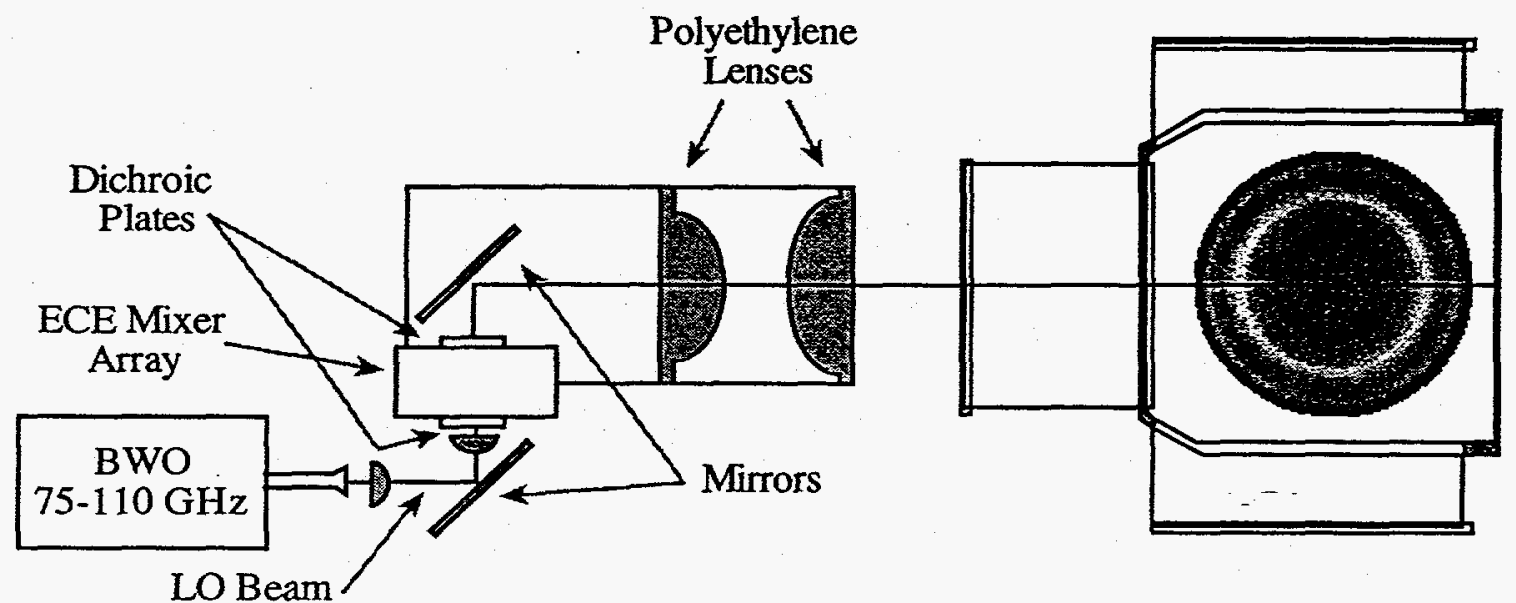

Fig. 1. ECE imaging implementation on TEXT-U. 
The ECE Imaging Array system has clearly shown itself to be capable of monitoring bulk temperature and temperature fluctuations. Figure 2 shows ECE Imaging Array data during a period of heavy MHD activity, where the temperature fluctuations due to MHD can be compared to the signal from one of the MHD channels. Figure 3 shows similar ECE Imaging Array data collected during a period of heavy sawteething activity.

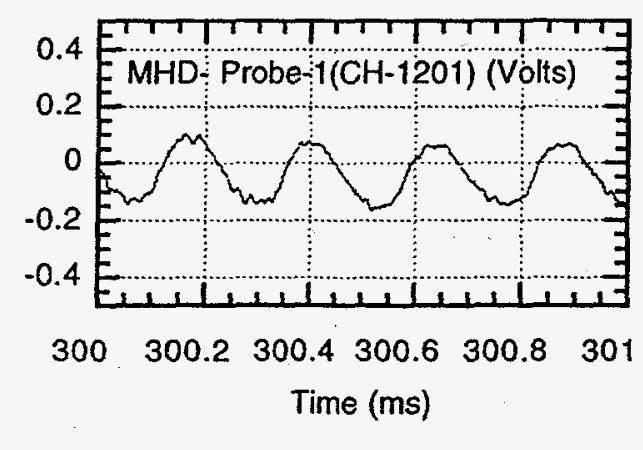

(a)

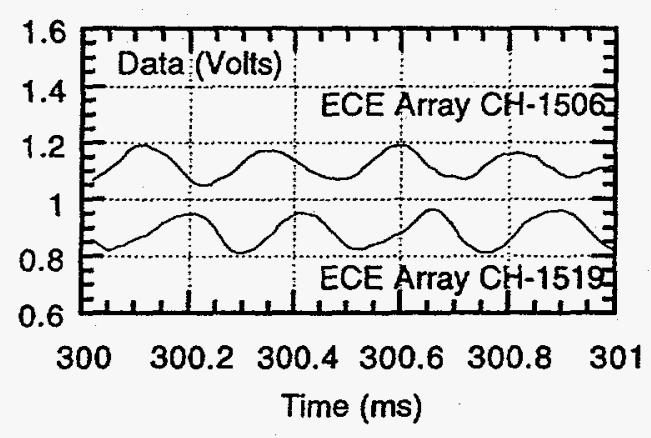

(b)

Fig. 2 ECE imaging array results from TEXT-U, showing data (SHOT 225198) from a TEXT-U MHD coil signal and two of the ECE imaging array channels.
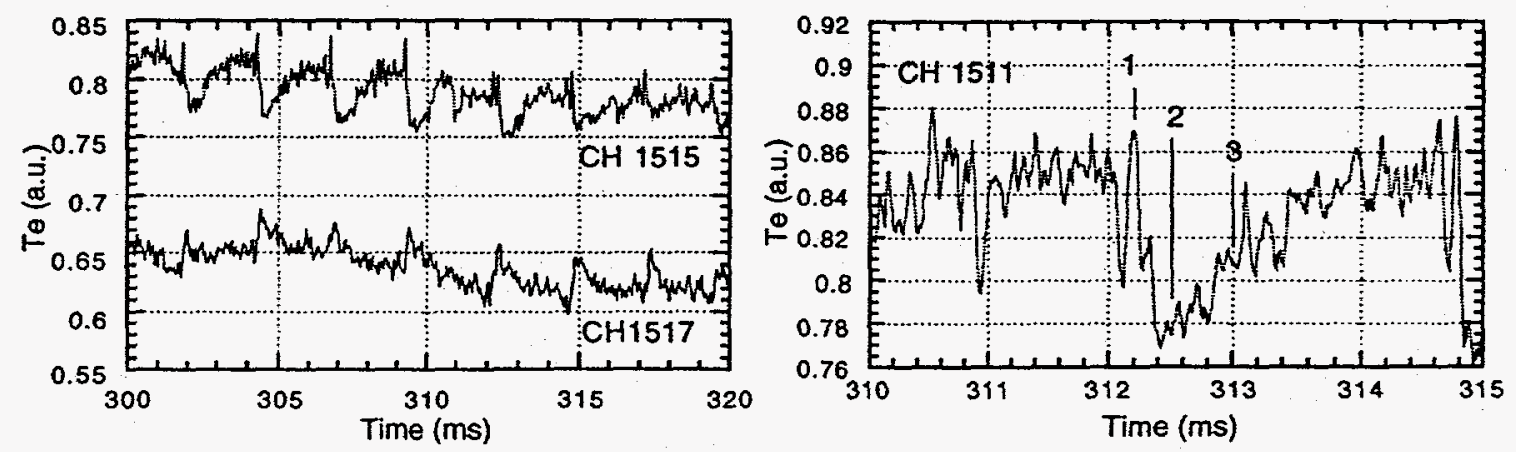

Fig. 3 ECE imaging array results from TEXT-U, showing data (SHOT 225465) from (a) a pair of array channels located on either side of the inversion radius, and (b) a closeup view in time of one the array signals. 
The spot size within the plasma for the TEXT-U ECE imaging optics has been experimentally determined and varies from channel-to-channel over a $1.0-1.7 \mathrm{~cm}$ range, with an interchannel spacing of $1.1-1.5 \mathrm{~cm}$. This extremely tight spot size proved to be crucial to our making the first ever detailed poloidal coherence measurements of electron temperature fluctuations. Shown in Fig. 4 are cross-correlation data from channels located $1.3 \mathrm{~cm}, 2.7 \mathrm{~cm}$ and $4.0 \mathrm{~cm}$ apart. The data is consistant with that observed by FIR collective scattering, and yields a poloidal wavenumber $\mathrm{k}_{\theta}$ of $1.6-2.0 \mathrm{~cm}^{-1}$ at $100 \mathrm{kHz}$, giving a phase velocity of $3.1-3.9 \times 10^{5} \mathrm{~cm} / \mathrm{s}$ which agrees with $v_{\mathrm{E} \times \mathrm{B}}+\mathrm{v}_{\mathrm{e}}^{*} \approx 3 \times 10^{5} \mathrm{~cm} / \mathrm{s}$.
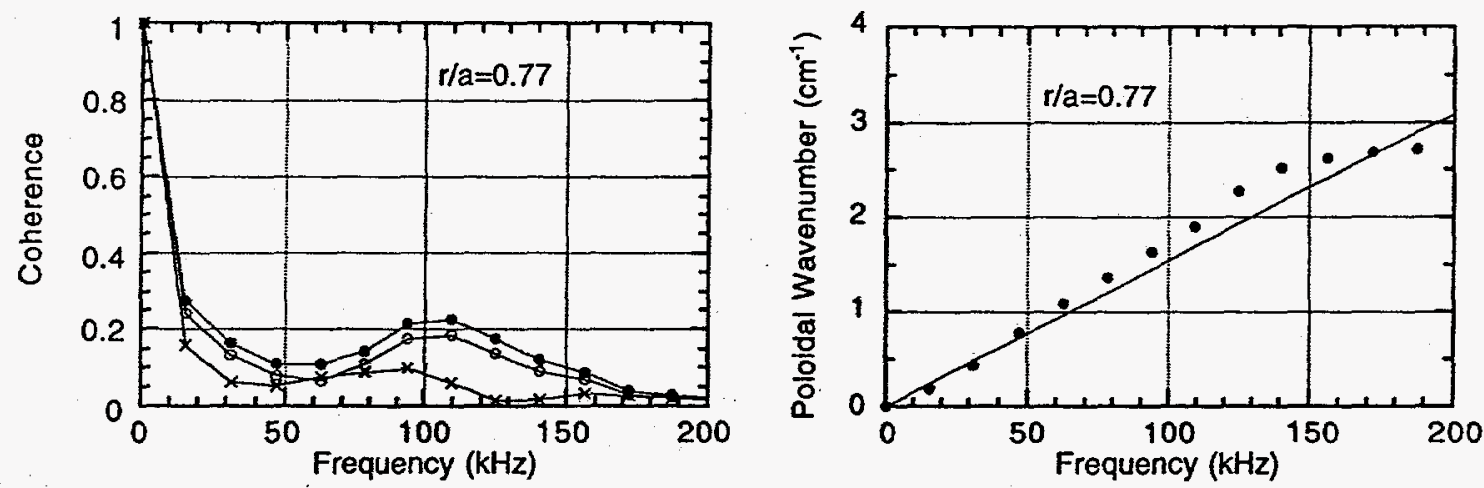

Fig. 4 Cross-correlation measurements between ECE imaging channels located $1.3 \mathrm{~cm}$ $(\bullet), 2.7 \mathrm{~cm}(\mathrm{o})$ and $4.0 \mathrm{~cm}(\times)$ apart.

In addition to the afore-mentioned fixed frequency LO measurements, the system was also operated in a high speed sweep mode. Here, the frequency of the LO source was swept over a 90-117.4 GHz frequency range, with a complete sweep and hence 2-D temperature profile obtained every $800 \mu \mathrm{sec}$. Analysis of this 2-D is ongoing, and will be presented along with the 1-D fluctuation data at the 11th Topical Conference on High Temperature Plasma Diagnostics to be held in Monterey, CA on May 12-16, 1996.

Design of a low cost waveguide-mounted second harmonic mixer array for 2-D FIR interferometry/polarimetry was initiated in FY95. The waveguide mixers will utilize an anti-parallel configuration of T/O beam-lead Schottky diodes (identical to those utilized in our hybrid ECE imaging arrays) placed within the RF waveguide. Key to the low cost nature of the mixer is the probe coupler built into the mixer, which samples only a small fraction of the LO power passing through the LO waveguide. This coplanar waveguide probe coupler has been extensively optimized utilizing Hewlett Packard's 3-D electromagnetic High Frequency Structure Simulator (HFSS) program. In array form, the mixers are placed end-to-end to form a chain of mixers with a common LO waveguide path. This allows for maximum LO utilization with the minimum of millimeter-wave 
components. Fabrication of a single channel mixer will commence once the mixer design has been completed, with fabrication and testing to be completed in FY96.

\section{Frequency Multipliers and Frequency Multiplier Arrays}

A number of technical improvements have been made in the area of frequency multiplier arrays that should result in the promise of $>30 \%$ conversion efficiencies form Ka-band to W-band. Superlattice barriers have been employed to reduce the leakage current of the SQBV devices which limited the device cut-off frequency as well as the tripling conversion efficiency of the Co-Principle Investigator's previous work in this area. New fabrication procedures developed at UC Davis provide significantly better diode isolation and reduce wafer surface loss; a factor of four improvement in the maximum efficiency is predicted from this step alone. A multiplier test fixture (see Fig. 5) has been designed and is under construction, which employs overmoded waveguide structures to reduce the diffraction loss associated with the previous quasi-optical configuration. An additional factor of 3-4 improvement in the maximum efficiency is predicted from the new test fixture. In addition, a recently developed high efficiency cooling system should permit $\mathrm{CW}$ operation at the $200 \mathrm{~W}$ input power level and corresponding high average power dissipation $(>100 \mathrm{~W})$.

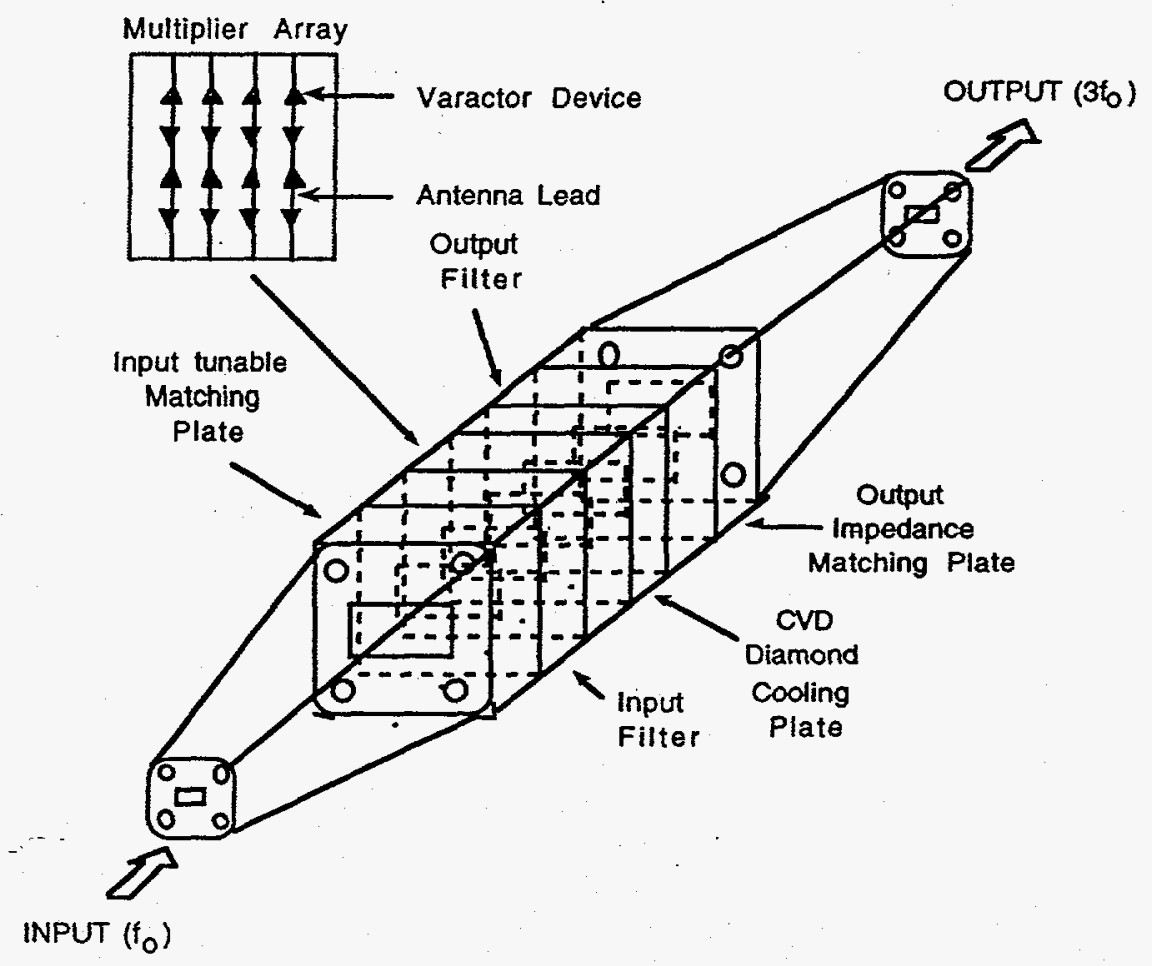

Fig. 5 Multiplier fixture 
Extensive system and device simulation studies have also been performed. The HP HFSS and Ansoft Maxwell 3D EM simulation codes have been employed to perform layout level optimization. An accurate simulation model for quasi-optical diode grid arrays has been developed using the actual device and antenna geometries thereby properly accounting for parasitics. The HP MDS and EESOF Libra software packages have been employed to perform harmonic balance simulation. Here, we note that the simulation results match well with our previous experimental results.

Designs have been developed for an initial proof-of-principle tripler system with $>30 \%$ efficiency and $>3 \mathrm{~W}$ output power at W-Band with a tunable bandwidth of $80-110 \mathrm{GHz}$ using a conventional $10 \mathrm{~W}$ Ka-Band TWT as the driver. Eventual systems could emply a microwave power module (MPM) driver (100 W output power) and two tripler arrays to produce $>15 \mathrm{~W} \mathrm{cw}$ output power at W-Band, and to offer dramatic savings in size, weight and cost as compared to conventional coupled-cavity TWT sources.

\section{Monolithic Beam Control Arrays}

A collaboration with Martin Marietta Electronic Systems was initiated to help develop a large variety of millimeter-wave devices. Our first collaborative effort involved the design, fabrication and characterization of multiple grid phase-shifting arrays for use in millimeterwave beam switching, steering and beam focusing/defocusing applications. As part of this collaboration, one of our students worked closely with Martin Marietta engineers in the design and fabrication of entire beam control arrays. The arrays were fabricated in their top-of-the-line MMIC fabrication facilities, and have the capability of being used as extremely fast switches, or as electronically-controlled beam steering and beam focusing/defocusing devices. Their light weight and compact size makes them ideal for use in space based radar, planetary mapping and resource systems.

Measurements with these BCAs have focused on Ka-band (26-40 GHz) and V-band (50-75 GHz), with both single and stacked grid pairs placed in an overmoded waveguide configuration. In transmission mode, a measured ON/OFF ratio of $41 \mathrm{~dB}$ is obtained at $62 \mathrm{GHz}$, (minimum insertion loss: $4.2 \mathrm{~dB}$ ) for the stacked grid pair, and a bandwidth of $\sim 6 \mathrm{GHz}$, with an average contrast ratio of $30 \mathrm{~dB}$ (see Fig. 6(a)). In reflection mode, a reflected beam phase shift of $-130^{\circ}$ has been experimentally obtained, with an average reflection loss of $2.7 \mathrm{~dB}$ for a single grid (as shown in Fig. 6(b)). Similar measurements at $38 \mathrm{GHz}$ have produced a reflected phase shift range of $170^{\circ}$ with $<4.5 \mathrm{~dB}$ loss. 

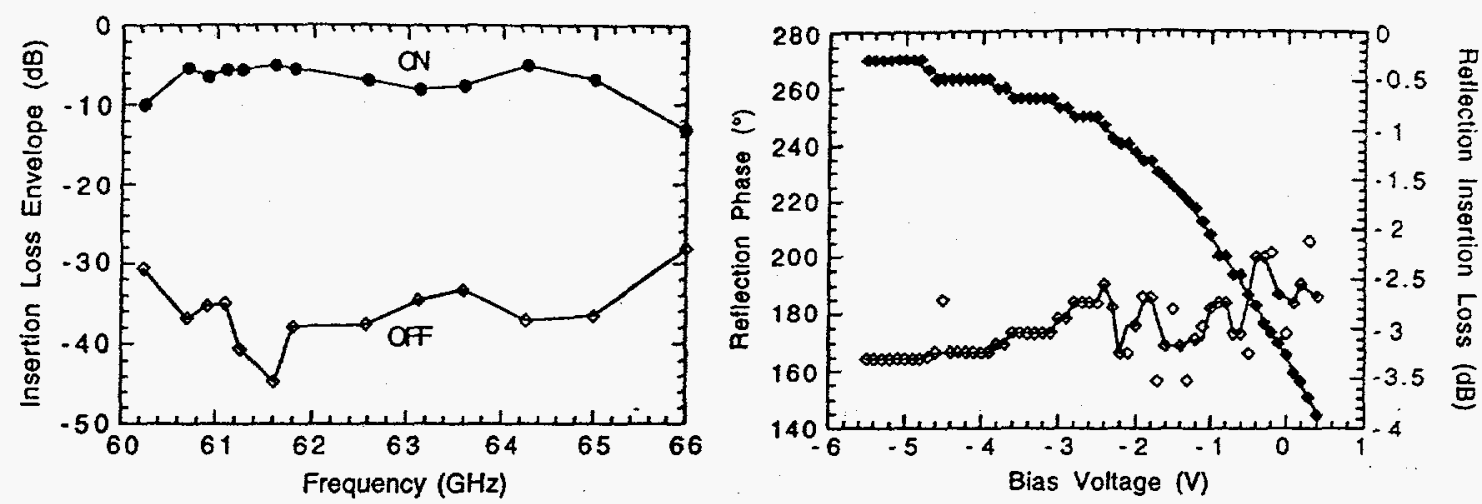

Figure 6. (a) Transmission switch results for a stacked grid pair of beam control arrays, and $(b)$ reflected beam phase shifter phase $(\diamond)$ and insertion loss $(\vartheta)$ results, showing a flat amplitude $130^{\circ}$ phase range (average insertion loss: $2.7 \mathrm{~dB}$ ).

A new method of stacking gridded beam control arrays (BCAs) has been designed. Although single grid reflection measurements produced record results, our two grid measurements failed to generate an increased phase range over the single grid (with tuner) configuration. After careful analysis, it is now believed that the failure of the two grid measurements is a direct consequence of diffraction loss in the resonator formed by the two GaAs grids. The new design calls for the wafers to be bonded together to eliminate the small gaps between the gridded wafers in the present test setup. This bonding can be done using facilities available at UC Davis. Simulations show that the new setup should be capable of $360^{\circ}$ phase range operated in transmission mode using a four grid system, while a $360^{\circ}$ phase range should be possible in reflection mode using a three grid system. Numerous application areas exist for such a transmission-mode phase shifter, including use as high speed phased array radar beam steerers for millimeter-wave imaging.

Opto-electronic beam control arrays (OBCAs) have also been fabricated. They are designed to function in a similar manner to the electronically-controlled BCAs except that the impedance properties of the Schottky photodiodes are controlled by the incident light intensity rather than by electrical bias signals. These arrays will also be tested in FY96 in the same $38 \mathrm{GHz}$ setup as the electronically-controlled BCAs, utilizing a $100 \mathrm{~W}$ pulsed diode laser array $(\lambda=800 \mathrm{~nm})$ to optically bias the arrays.

\section{Nonlinear Transmission Lines and Nonlinear Delay Lines}

Monolithic nonlinear transmission lines (NLTLs) are under investigation for use in ultra-wideband radar imaging, time domain materials characterization and magnetic fusion plasma applications. Considerable progress has been made in the past quarter in modeling 
the devices and circuits that are utilized in our monolithic NLTLs and in our monolithic frequency multiplier, beam control and imaging arrays. Extensive work has been conducted on modelling the diode and lead layout with Hewlett Packard's 3-D electromagnetic High Frequency Structure Simulator (HFSS) program. A new simulation model has been developed by one of the Co-Principal Investigator's Ph.D. students, which allows for the inclusion of device resistive layers directly into the device layout. This model is capable of layout level optimization, such as reducing parasitic capacitance, reducing series resistance and improving device cutoff frequency. Output parameters obtained from the EM model are fed into HP's Microwave Design System (MDS) program to obtain an equivalent circuit model which can be used to more accurately simulate the NLTLs. Figure 1 shows the cross sectional view of a back-to-back diode structure, utilized in our monolithic NLTLs and quasi-optical frequency multiplier arrays.

Monolithic NLTLs have been fabricated that have compressed $\sim 100$ psec risetime pulses into directly measured $<37$ psec risetime pulses with $\approx 12 \mathrm{~V}$ peak voltages. Deconvolving out the instrument function of the digital sampling oscilloscope utilized to take the measurement, however, indicates that short pulses with $<28$ psec risetimes and peak voltages of $\approx 15 \mathrm{~V}$ were obtained (see Fig. 7), in good accordance with numerical simulations (which predict $\sim 25$ psec risetime pulses with $\sim 16 \mathrm{~V}$ peak voltages) using measured device parameters.

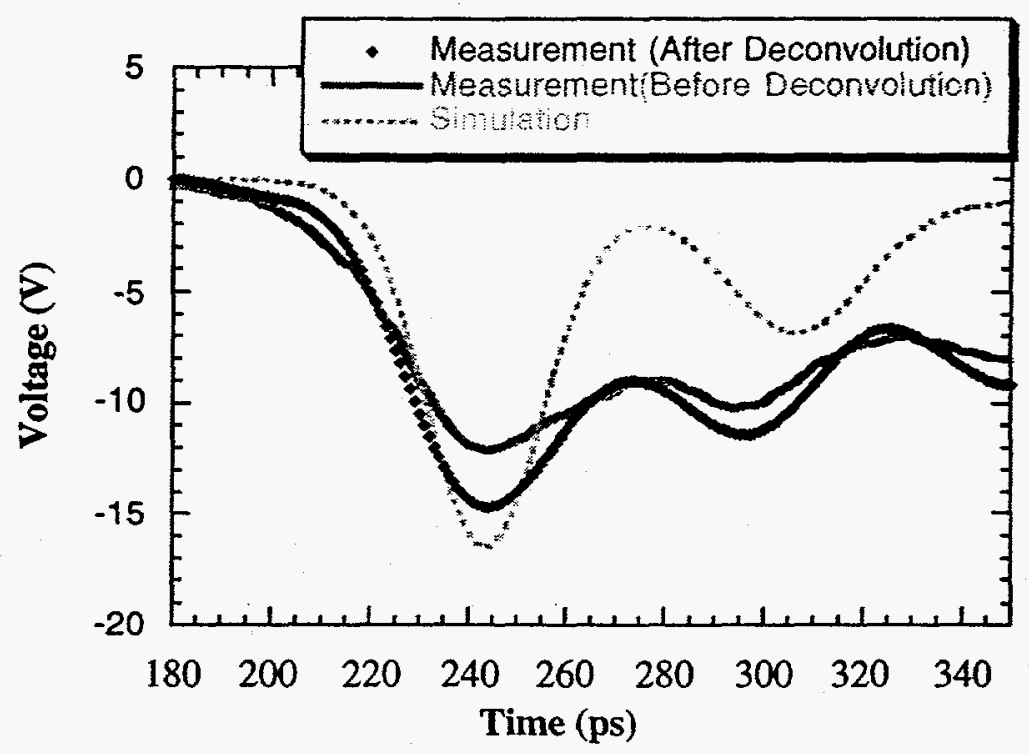

Figure 7. Output from a nonlinear transmission line (measurement and simulation).

A proof-of-principle wideband radar system is currently under development with the eventual goal of developing a low cost collision avoidance radar system. This extends the 
LLNL Micropower Impulse Radar technology by approximately a factor of 10-100 in frequency. An impulse driver, timing circuitry and NLTL compressor circuit will eventually be packaged together into one unit which will form the radar transmitter component. The radar receiver will be composed of a sampling circuit, nonlinear delay line (NDL) and differential amplifier circuitry. Work on this project to date has produced a hybrid sampling circuit that was designed, built and utilized to successfully reconstruct an input pulse waveform. A differential amplifier circuit has been designed in order to integrate the output signals from the sampling circuit, and work has now begun on designing a monolithic nonlinear delay line (NDL) circuit to provide voltage-controlled true time delay to the afore-mentioned sampling circuit. 


\section{Advanced Photoinjector Laser Technologies}

Work in this research area is involved with the development of short pulse, high brightness laser-driven guns, multi-MW high and the development of a completely new "shoe-box" sized laser concept which incorporates a mode-locked semiconductor laser, a diode laser pumped Li:SAF amplifier and a CsTe photocathode. This work has been the subject of 3 Physical Review Letters, a Physical Review E, an Applied Physics Letters, a Physics of Plasmas and a Nuclear Instruments and Methods in Physics Research publications.

A state-of-the-art X-band $(-8-11 \mathrm{GHz})$ if gun is under development as an RF photoinjector source aimed at producing psec high brightness electron bunches for advanced accelerator and coherent radiation generation studies. The major components of the photoinjector system are the RF drive subsystem, including the $20 \mathrm{MW} X$-band klystron and modulator, the laser subsystem, including the solid state oscillator, multipass amplifier, and frequency multiplier, and the accelerator structure. Progress in each of these areas is addressed separately below.

For the RF drive subsystem, the pulse transformer circuit and tank have been built, delivered and installed. A state-of-the-art SLAC klystron has been acquired and loaded into a pulse transformer tank. During the loading process, the vacuum integrity of the klystron was tested and proved satisfactory, and the filament was found to be in good working condition as well. The RF drive cooling system has been built and tested, meeting all SLAC flow rate specifications for the klystron and solenoid. All vacuum compatible hardware has been installed and cold tested, and the step-up transformer installed and all preliminary tests completed. The driver TWTA itself was taken to SLAC and tested. The parameters measured were approximately $900 \mathrm{~W}$ out with $10 \mathrm{~mW}$ drive at $8.548 \mathrm{GHz}$, well within the drive requirements for saturated output.

The 1-1/2 accelerator cell structure cold test cavity design is complete, and fabrication is underway (cutting metal). The RF gun is driven by a high repetition rate $(2.214 \mathrm{GHz}$ ) AlGaAs semiconductor laser oscillator and eight-pass Ti-Sapphire amplifier system which is complete. The entire RF gun facility is expected to be operational by the end of FY95.

An $\mathrm{AlGaAs}$ quantum well semiconductor laser oscillator has been fabricated and operated at a wavelength of $830 \mathrm{~nm}$. Synchronous modelocking at $1.46 \mathrm{GHz}$ has been demonstrated with pulse widths as low as 20 ps (without the multiple quantum well saturable absorber). The optical bandwidth has been measured, with results indicating that transform-limited pulses as short as $150 \mathrm{fs}$ are possible with this oscillator ( $\sim 1 \mathrm{ps}$ obtained to date). Jitter performance of the laser is quite low, with $400 \mathrm{fs} r m s$ measured over $100 \mathrm{~s}$. 